\title{
L'Institut Francilien de Recherche sur les Atomes Froids (IFRAF), un réseau de recherche de pointe soutenu par la Région île-de-France
}

\section{Michèle LEDUC}

Directrice de I'Institut Francilien de

Recherche sur les Atomes Froids (IFRAF)

Laboratoire Kastler-Brossel, ENS

leduc@lkb.ens.fr

\section{Un domaine scientifique d'une exceptionnelle vitalité}

C'est en 2004 qu'est née l'idée de rassembler toutes les équipes travaillant sur les atomes froids dans la région parisienne, ce domaine avait déjà une vingtaine d'années d'existence et se développait à vive allure dans le monde entier. Le Laboratoire Kastler Brossel (le LKB) à l'École normale supérieure (ENS) y a joué un rôle de pionnier pour la France.

Autour de Claude Cohen-Tannoudji, professeur au Collège de France, s'est constituée une équipe regroupant Jean Dalibard, Christophe Salomon, Alain Aspect, qui tous ensemble se sont lancés dans le domaine durant les années 80 . Le renouveau spectaculaire de la physique atomique apporté par les atomes froids attira de plus en plus de jeunes brillants chercheurs à l'ENS, où se constitua bientôt une école de recherche réputée, très en prise avec les groupes du monde entier investis dans le même domaine. Le concept du piège magnétooptique y fut élaboré, bientôt adopté comme l'outil universel pour les gaz refroidis et piégés par la lumière laser ; les températures anormalement basses qui y étaient mesurées trouvèrent une explication. Le domaine connut une véritable explosion après 1995 quand la condensation de Bose-Einstein fut enfin réalisée expérimentalement aux ÉtatsUnis, puis ensuite en France. Et le prix Nobel octroyé à Claude Cohen-Tannoudji en 1997, en même temps qu'à ses amis Bill Phillips et Steven Chu, augmenta encore le prestige du domaine.

Pourtant la recherche sur les atomes froids ne resta pas cantonnée à l'ENS. Alain Aspect quitta le LKB en 1993 pour se lancer dans l'aventure à Orsay, rejoint par son élève Philippe Grangier avec lequel il créa un nouveau laboratoire à I'Institut d'Optique. De nombreux jeunes chercheurs formés à l'école de Claude Cohen-Tannoudji commencèrent à essaimer vers d'autres établissements, pour beaucoup dans la région parisienne. Ainsi, Pierre Pillet rejoignit le Laboratoire Aimé Cotton à Orsay, Hélène Perrin s'installa au Laboratoire de Physique des Lasers à Paris-Nord, et d'anciens doctorants du LKB comme Pierre Lemonde ou Franck Pereira-dos-Santos allaient à l'Observatoire de Paris développer des instruments de métrologie à atomes froids. Tous ces jeunes chercheurs issus de l'ENS développèrent à leur tour des équipes vers lesquelles affluèrent de très nombreux doctorants et des postdoctorants issus de nombreux pays, tout particulièrement d'Allemagne. La communauté francilienne était suffisamment petite pour que tous les chercheurs permanents se connaissent et suffisamment concentrée en île-de-France pour que la communication s'installe facilement entre les équipes.

\section{Le regroupement : une initiative opportune portée par la Région}

C'est alors qu'est née l'idée de regrouper en réseau toutes ces équipes, expérimentales mais aussi théoriques, afin de leur donner des moyens de travailler ensemble en bonne synergie. Un excellent modèle était $d$ 'ailleurs fourni par

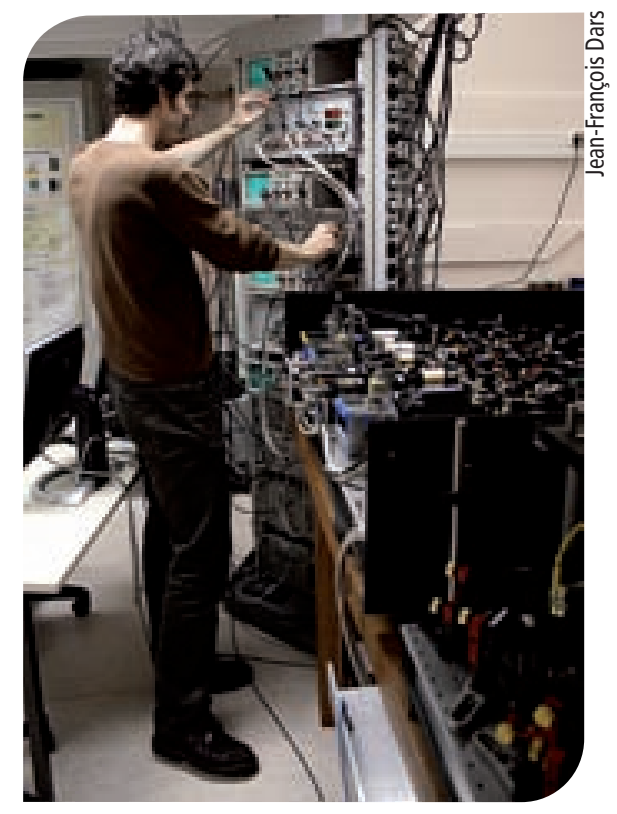

Une expérience d'atomes froids au Laboratoire Kastler-Brossel.

le Center for Ultracold Atoms (le CUA, présidé alors par Daniel Kleppner), rassemblant les équipes de MIT et Harvard impliquées dans les recherches avec des atomes froids. Les échos des succès du CUA parvenus en France faisaient rêver les équipes franciliennes, alors à la peine pour rassembler les financements nécessaires à leurs recherches de pointe. Une ambition de même nature s'est alors imposée pour regrouper les équipes du même domaine actives à Paris et dans sa région. Le projet qui a vu le jour en 2004, après plusieurs remaniements, a bénéficié des encouragements de Yvan Chémama, à l'époque responsable du secteur recherche pour le Conseil régional de l'île-de-France.

Présenté par Claude Cohen-Tannoudji devant le Conseil Scientifique de la Région, le projet a été approuvé par les instances politiques régionales. II comporte évidemment un important volet 
de recherche très fondamentale mais il débouche aussi sur des applications très concrètes dans le domaine de l'instrumentation - ce qui est cohérent avec les objectifs de la Région en matière d'innovation. Un contrat d'une durée de cinq ans a finalement été signé entre les responsables de la Région et les six établissements universitaires auxquels appartiennent les équipes du réseau, qui s'est baptisé, puis fait connaître avec son logo rouge, sous le nom d'IFRAF. Le Conseil régional I'a reconnu comme un « Domaine d'Intérêt Majeur » (DIM), avec 13 autres DIM dans des secteurs scientifiques différents. Le total des financements régionaux prévus par le contrat de I'IFRAF pour la période 2005-2010 était de $10 \mathrm{M} €$, impliquant un cofinancement par d'autres sources de crédits. Ces financements sont prolongés jusqu'en 2012, en attendant les décisions relatives aux années suivantes.

\section{Un ensemble de 200 chercheurs travaillant en concertation}

L'IFRAF est un réseau de 33 équipes de recherche situées en île-de-France et réparties sur différents sites. Il a été fondé par sept établissements : le CNRS, l'École normale supérieure, l'Observatoire de Paris, I'Institut d'Optique Graduate School, et les Universités UPMC, ParisSud 11 et Paris 13. Les équipes de I'IFRAF appartiennent à six laboratoires tous associés au CNRS :

- le Laboratoire Kastler-Brossel (LKB) à Paris

- le Laboratoire des SYstèmes de Référence Temps-Espace (SYRTE) à l'Observatoire de Paris

- le Laboratoire Charles Fabry à I'Institut d'Optique (LCFIO) à Palaiseau

- le Laboratoire Aimé Cotton (LAC) à Orsay

- le Laboratoire de Physique Théorique et Modèle Statistiques (LPTMS) à Orsay

- le Laboratoire de Physique des Lasers (LPL) à Villetaneuse.

L'IFRAF s'est également associé, depuis sa création, à huitéquipes situées dans d'autres établissements, les Universités Paris

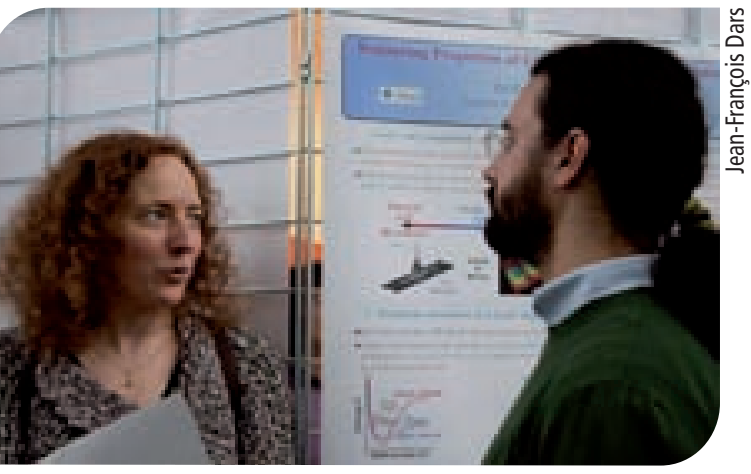

chercheurs des six laboratoires) et qui comporte aussi des membres extérieurs issus du monde industriel. Ce Directoire joue le rôle d'un Conseil Scientifique choisissant les projets et définissant les orientations stratégiques de l'Institut. Ses décisions sont ensuite discutées par son conseil d'administration. Le mode de travail choisi par I'IFRAF est du type « bottom-up ", c'est-à-

Workshop IFRAF à Palaiseau (2009).

Diderot et Cergy-Pontoise pour l'île-deFrance. L'ensemble des chercheurs de I'IFRAF comporte 75 permanents (enseignants-chercheurs ou chercheurs-CNRS) et beaucoup de jeunes chercheurs, doctorants et post-doctorants (environ 120) dont le nombre varie légèrement d'une année sur l'autre. Ces jeunes chercheurs, surtout les postdoctorants, sont de toutes les nationalités, avec un grand nombre d'Allemands et d'Italiens mais aussi des Russes, des Chinois et des Indiens.

La structure administrative de I'IFRAF est légère : c'est un Groupement d'Intérêt Scientifique (GIS). Son siège est situé à l'École normale supérieure, qui est l'établissement support habilité à recevoir et gérer les financements en provenance de la Région. Son fonctionnement est fondé sur les travaux du Directoire, une assemblée représentative des utilisateurs (les

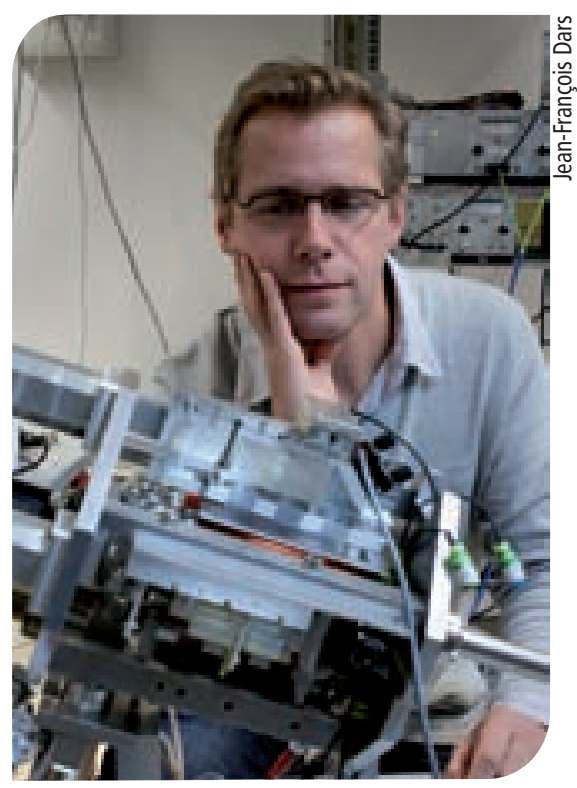

Arnaud Landragin et le gyromètre à atomes froids au Syrte. dire qu'il examine des projets de recherche qui émanent de ses différentes équipes, sans s'inscrire dans une programmation thématique imposée : en cela l'IFRAF se rapproche du fonctionnement de la NSF (National Science Fondation) aux États-Unis ou encore du programme dit « blanc » de l'ANR (Agence Nationale de Recherche, en place depuis 2006 en France).

\section{Des moyens accrus pour des projets en commun}

L'objectif majeur de l'IFRAF est de développer la coopération entre ses équipes, de façon à augmenter leur productivité scientifique dans un paysage mondial de plus en plus compétitif. Le travail en synergie évite souvent une perte de productivité liée à des essais en parallèle sans concertation. Les développements instrumentaux et les astuces de montage circulent de groupe en groupe, évitant la perte de temps et parfois d'argent. Des projets en commun entre plusieurs laboratoires qui n'auraient certainement pas vu le jour sans l'existence de I'IFRAF, peuvent être décidés. Ainsi, depuis sa création, cinq nouvelles expériences ont été démarrées à partir de zéro en associant plusieurs équipes appartenant à deux voire trois - laboratoires de l'institut. Sa flexibilité et sa réactivité sont d'autres avantages de I'IFRAF, lui permettant de décider de se lancer sur un sujet dont l'importance pour la recherche vient juste d'émerger, sans prendre de retard par rapport à la concurrence à l'étranger. L'apport financier des moyens engagés par la Région île-de-France dans I'IFRAF est évidemment un élément essentiel de sa réussite. II est utilisé pour $\gg$ 
accroître le nombre des jeunes chercheurs, doctorants et postdoctorants, mais aussi pour inviter des visiteurs étrangers qui viennent travailler quelques semaines ou quelques mois dans ses laboratoires.

Les financements régionaux servent aussi beaucoup à des investissements. Ils permettent de rénover certaines infrastructures vieillissantes ou de construire de nouveaux locaux. Mais il sont surtout un formidable levier pour les équipements des expériences d'atomes froids. Ces expériences sont extrêmement complexes et assez demandeuses de matériel de toute sorte (optique, lasers stabilisés, ultra-vide, électronique de puissance, informatique de course, etc.) : I'ensemble de l'appareillage d'une expérience typique représente un budget compris entre 0.5 et $1 \mathrm{M} €$ et occupe une pièce entière. Les appareillages une fois construits servent entre cinq et dix ans à des projets successifs par modification progressive des montages existants. Mais I'activité la plus notable de l'IFRAF est la construction de cinq nouvelles expériences impliquant obligatoirement la participation de plusieurs équipes dans différents domaines qui ont déjà donné des résultats fructueux. Dans bien des cas, I'impulsion initiale donnée par I'IFRAF a engendré ensuite d'autres financements plus importants, illustrant l'effet levier de crédits d'importance modérée mais bien placés au bon moment.

\section{Les grandes thématiques actuelles}

$\mathrm{Si}$, comme il a été dit plus haut, les projets scientifiques de l'IFRAF proviennent des idées suggérées par les équipes, il se trouve néanmoins qu'ils peuvent être regroupés selon trois grands axes correspondant aux grandes tendances actuelles du domaine des atomes froids qui ne cesse de s'amplifier.

- Les gaz quantiques et les comportements collectifs dans les condensats de Bose-Einstein. Les atomes refroidis à des températures ultra-basses sont soit des bosons, soit des fermions. Dans les deux cas, les tendances les plus promet-

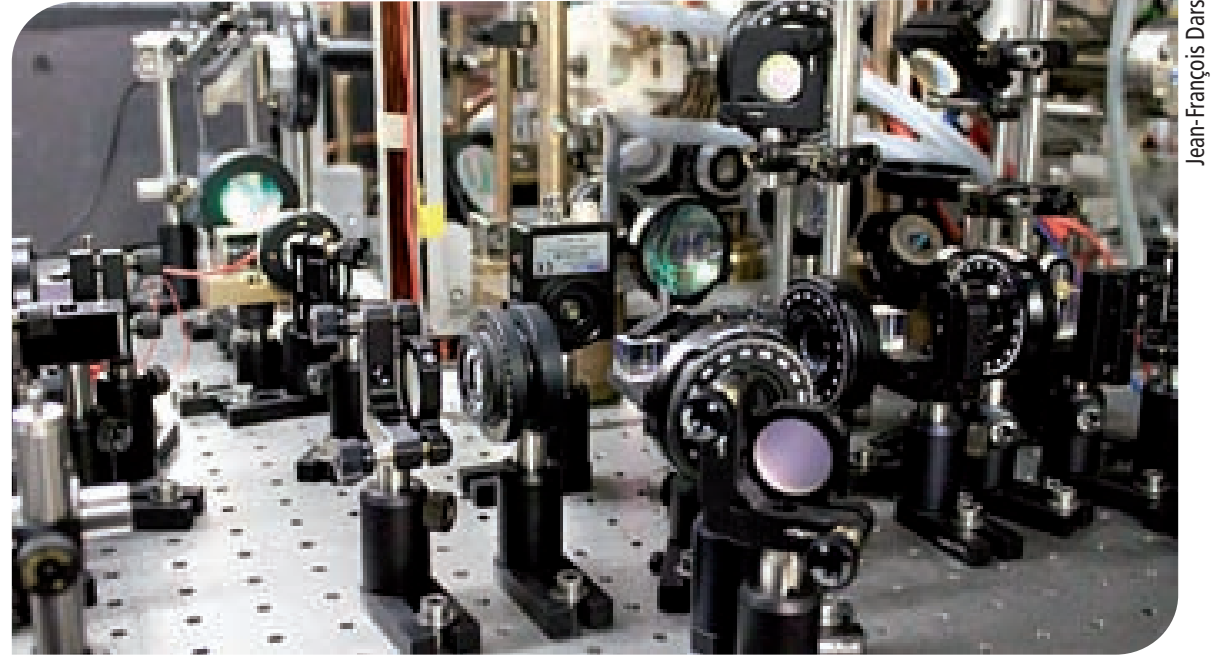

Table d'optique pour une expérience d'atomes froids.

teuses consistent à insérer ces gaz froids dans des potentiels périodiques (ou désordonnés) créés par des ondes lumineuses. On réalise ainsi des systèmes modèles pour la matière condensée : les atomes fermioniques piégés ainsi présentent beaucoup d'analogies avec les électrons dans un cristal par exemple, avec cet avantage que les paramètres des réseaux optiques avec les atomes froids sont modifiables à volonté et que l'on peut appliquer des méthodes subtiles pour modifier, avec des champs magnétiques, les interactions entre les atomes. De plus, on peut choisir la géométrie de ces réseaux et surtout travailler en deux dimensions. De nouvelles phases sont prédites et devraient être observées. On espère, à terme, une meilleure compréhension de phénomènes tels que la superfluidité et la supraconductivité, ou encore l'apparition du ferromagnétisme et de I'anti-ferromagnétisme. Une nouvelle voie se développe en parallèle avec le refroidissement de molécules à partir de deux ou trois atomes froids entrant en collision, ou bien pour des molécules plus complexes en utilisant des techniques issues de la physique des accélérateurs.

- La métrologie et l'instrumentation. L'utilisation des atomes froids fait gagner plusieurs ordres de grandeur sur la précision des horloges, des gyromètres, des gravimètres et des capteurs inertiels pour le positionnement et la navigation. La miniaturisation de ces instruments sur des puces à atomes est un enjeu impor- tant dans la perspective des applications spatiales et du positionnement par satellite. L'IFRAF a lancé plusieurs expériences nouvelles, I'une relative à une horloge sur puce (projet Chip-Clock) et l'autre à un gyromètre atomique perfectionné (projet BIARO), ainsi qu'un projet technologique (MINIATOM) avec des industriels pour des capteurs inertiels miniaturisés pour l'espace (voir l'article de P. Bouyer dans ce numéro). Notons aussi que les équipes de l'IFRAF sont impliquées dans la réalisation du projet PHARAO qui consistera à placer une horloge à atomes froids dans l'espace, destinée, entre autres, à des tests de relativité générale. - L'information quantique. Les gaz d'atomes froids et ultra-froids, de même que les atomes ou ions isolés et piégés en petit nombre, permettent un niveau de contrôle sans précédent sur les degrés de liberté quantiques de ces systèmes et I'on peut déjà envisager de nouveaux dispositifs qui exploitent les effets quantiques fondés sur l'intrication entre particules (photon/photon, atome/photon ou atome/atome). Les ions piégés sont une autre option. On sait maintenant réaliser des générateurs de photons uniques, des répéteurs. Certaines applications ont déjà émergé, comme la cryptographie quantique. Pour d'autres le domaine est encore prospectif. L'IFRAF a démarré deux nouvelles expériences dans ce domaine : le projet MICROBEC (mini-condensats dans un réseau optique) et un montage d'atomes de Rydberg intriqués. 


\section{Rayonnement de I'IFRAF}

En cinq années d'existence l'IFRAF s'est progressivement fait connaître et reconnaître à travers ses multiples actions de formation des jeunes et en direction du grand public. Chaque année a lieu une session de formation des jeunes doctorants dans le domaine des atomes froids, avec le soutien de I'IFRAF, à l'École Internationale de Physique des Houches. En outre, I'IFRAF, depuis sa formation, a subventionné de nombreux " workshops » internationaux, en île-de-France mais aussi aux Houches et à l'étranger, auxquels ont assisté des jeunes chercheurs de I'IFRAF, ce qui leur a permis de nouer des contacts pour le long terme. Ainsi se sont progressivement tissées des relations privilégiées avec plusieurs centres de recherche étrangers investis dans les mêmes domaines que l'IFRAF. Des conventions de coopération tripartites sont maintenant signées entre IFRAF, QUEST et ACQAO. QUEST (Center for Quantum Engineering and Space-Time Research) est le centre d'excellence constitué autour de I'Université de Hanovre; ACQAO (Australian Center for Quantum Atom Optics) regroupe l'ensemble des laboratoires du domaine en Australie. Les trois instituts organisent des échanges de jeunes chercheurs et collaborent sur des projets communs.

L'IFRAF s'investit également beaucoup dans la diffusion des connaissances amassées par ses équipes auprès du grand public. De nombreux chercheurs se mobilisent pour des conférences publiques en s'adaptant au niveau requis: les bars des sciences, les émissions de radio, les interviews, les films de vulgarisation. Des clips sur les atomes froids sont en cours de réalisation avec le CNRS et seront bientôt en ligne sur le site de I'IFRAF. Fin 2009, des jeunes se sont mobilisés pour faire fonctionner, au Palais de la découverte, un piège magnéto-optique, monté au Laboratoire PHLAM de Lille. Cette exposition intitulée "Pris au piège " a duré deux mois dans le cadre de la série "Un chercheur, une manip" ». Et un jeune enseignant issu du LKB (E. Jahier, auteur d'un article dans ce numéro) vient d'écrire un livre rigoureux mais d'accès facile intitulé "Les atomes froids " chez EDP Sciences. Toutes ces activités en direction du public sont considérées comme très importantes par l'ensemble de I'IFRAF et devraient être amplifiées dans le futur.

Le rayonnement national et international de I'IFRAF va croissant depuis sa fondation, en 2005. L'IFRAF est maintenant partie prenante de tous les grands événements internationaux dans le domaine. Un des effets de sa renommée est qu'il a été choisi pour organiser, sur le campus de Polytechnique à Palaiseau, la prochaine conférence mondiale de physique atomique en 2012 (ICAP 2012), pour laquelle 700 participants environ sont attendus. Un élan exceptionnel a été donné en France au domaine des atomes froids grâce à la vitalité de I'IFRAF. II importe aujourd'hui de maintenir cet élan et de l'amplifier.

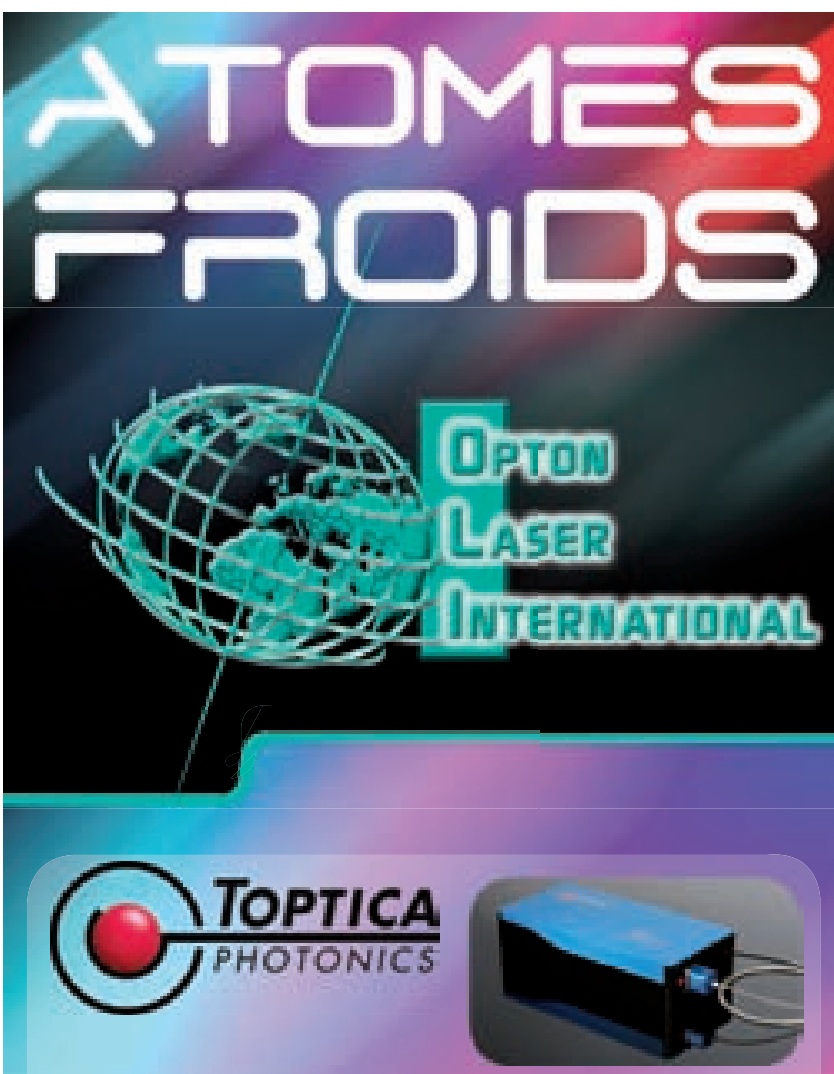

- Diodes laser en cavité externe Technologie pro, ultrastable : 780,850 , $940,1040 \mathrm{~nm}$ et $632-1770 \mathrm{~nm}, 370-489 \mathrm{~nm}$

- Systèmes amplifiés TA pro : 2W, 649-1083nm

- TA/FA pro - SHG / FHG : 205-590nm

- Lasers à fibre fs FFpro : 1560 nm + 780nm + supercontinuum

- Diodes laser FP avec ou sans traitement AR, diodes DFB, chips amplificateurs

- Isolateurs

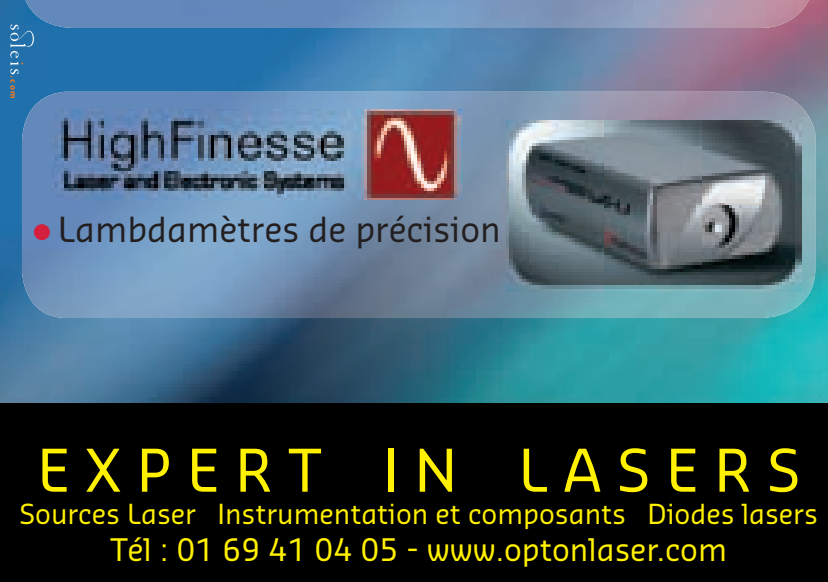

